\title{
Skin Testing for Suspected lodinated Contrast Media Hypersensitivity
}

Rik Schrijvers, MD, PhD ${ }^{a, b}$, Christine Breynaert, MD, PhD ${ }^{a}$, Yazid Ahmedali, MD ${ }^{b}$, Jean-Luc Bourrain, MD ${ }^{b}$, Pascal Demoly, MD, PhD ${ }^{\mathrm{b}, \mathrm{c}}$, and Anca Mirela Chiriac, MD ${ }^{\mathrm{b}}$ Leuven, Belgium; and Montpellier and Paris, France

What is already known about this topic? A subgroup of iodinated contrast medium (ICM) hypersensitivity reactions is immunologically mediated, potentially life threatening, and can be diagnosed using skin testing. Skin testing is preferred early (1-6 months) after the event. However, the negative predictive value of skin testing is insufficiently evaluated.

What does this article add to our knowledge? Skin testing for potential ICM hypersensitivity can identify safe alternative(s) for re-exposure, especially in patients with a history of an immediate hypersensitivity reaction. Reactions on reexposure are infrequent and mostly milder.

How does this study impact current management guidelines? Our work validates the role of skin testing to identify safe alternatives and offers an allergologist-driven, clinical history-, and skin-test-based approach to guide ICM reexposure, without the need for provocation testing outside an imaging context.

BACKGROUND: The management of iodinated contrast medium (ICM) hypersensitivity has been a matter of debate. Skin testing to identify a subgroup of ICM allergic patients has been proposed, in addition to complete avoidance, provocation testing, or premedication.

Objective: The objective of this study was to assess the negative predictive value (NPV) of skin testing for ICM.

METHODS: Patients with a hypersensitivity reaction to ICM who underwent skin testing during a 13.5-year period at a single center were evaluated for re-exposure to a negatively skin-tested ICM. Premedication, consisting of second-generation H1antihistamines twice a day 48 hours before the examination, was

\footnotetext{
a Laboratory of Clinical Immunology, Department of Microbiology and Clinical Immunology, KU Leuven, Leuven, Belgium

${ }^{\mathrm{b}}$ Exploration des Allergies, Département de Pneumologie et Addictologie, Hôpital Arnaud de Villeneuve, University Hospital of Montpellier, Montpellier, France 'Sorbonne Universités, UPMC Paris 06, UMR-S 1136, IPLESP, Equipe EPAR, Paris, France

RS is an EAACI research grant awardee, Fundamenteel klinisch mandaat FWO.

Conflicts of interest: C. Breynaert has received research support in the format of a C1 internal grant; and has received travel support for congresses. P. Demoly has received consultancy fees from Stallergenes Greer, ALK, Chiesi, Medarini, and ThermoFisher Scientific; has received research support from AstraZeneca and MEDA/Mylan; and has received lecture fees from ALK and Stallergenes Greer. A. M. Chiriac has received research support from COBIOPHAD (Horizon2020 Project). The rest of the authors declare that they have no relevant conflicts of interest.

Received for publication November 6, 2016; revised September 10, 2017; accepted for publication October 9, 2017.

Available online $\mathbf{\square}$

Corresponding author: Anca Mirela Chiriac, MD, Allergy Unit, Arnaud de Villeneuve Hospital, University Hospital of Montpellier, 371, Avenue du Doyen Gaston Giraud, Montpellier Cedex 5, Montpellier 34295, France. E-mail: a-chiriac@chu-montpellier.fr.

2213-2198

(C) 2017 American Academy of Allergy, Asthma \& Immunology

https://doi.org/10.1016/j.jaip.2017.10.040
}

advised only for patients with mast cell disorder or chronic urticaria who had negative skin tests.

RESULTS: A total of 597 patients tested for $\mathbf{4 2 3}(\mathbf{7 0 . 9} \%)$

immediate, 118 (19.7\%) nonimmediate, and 56 (9.4\%)

hypersensitivity reactions with undetermined chronology were included. Eighty (13.4\%) patients were skin test positive. Reexposure to ICM occurred in $233(39.0 \%)$ patients and was tolerated in 16 of $17(94.1 \%)$ with at least 1 positive skin test and 201 of $216(93.1 \%)$ with all negative skin tests. Reaction intensity was similar in 4, milder in 10, unknown in 1, and worse in 1 patient although this reaction was deemed to be nonallergic in hindsight. Premedication was administered in $20.7 \%$ of patients and associated with more reactions $(19.4 \%$ vs $5.7 \%, P=.01)$. The overall NPV of skin testing for ICM was $93.1 \%$ (95\% confidence interval $[\mathrm{CI}] \mathbf{8 9 . 1 \%}$ to $\mathbf{9 6 . 0 \%}$ ), and for immediate and nonimmediate hypersensitivity reactions $\mathbf{9 4 . 2 \%}$ (95\% CI $89.6 \%$ to $97.2 \%)$ and $86.1 \%$ (95\% CI $72.1 \%$ to $94.7 \%)$, respectively. We cannot exclude some challenges occurred with a different than the initial culprit ICM, possibly overestimating the NPV. CONCLUSIONS: Skin testing for potential ICM hypersensitivity can identify safe alternative(s) for ICM re-exposure especially in patients with an immediate hypersensitivity reaction and/or skin test-proven ICM drug allergy. Reactions on re-exposure were infrequent, mostly milder, and occurred in some patients despite premedication. (C) 2017 American Academy of Allergy, Asthma \& Immunology (J Allergy Clin Immunol Pract 2017; $\square:$ a-口)

Key words: Allergy; Hypersensitivity; Iodinated contrast media; Radiocontrast; Anaphylaxis; Negative predictive value; Skin tests; Drug allergy

Since their introduction in the 1950s, iodinated contrast media (ICMs) have been among the most commonly prescribed drugs for radiological imaging. ${ }^{1}$ Four structurally different groups can be distinguished (ionic or nonionic, monomeric or dimeric). ${ }^{2}$ 


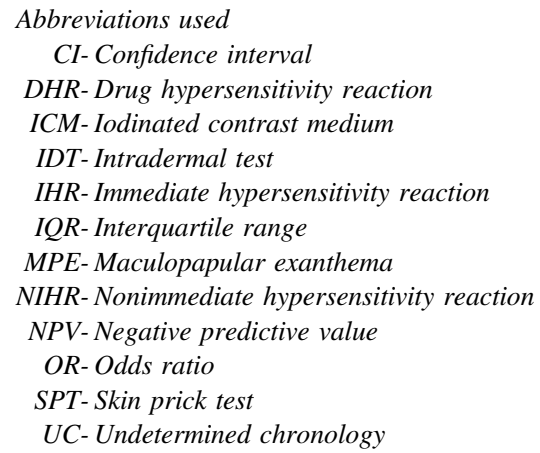

High-osmolarity ionic monomers (amidotrizoate and ioxithalamate) have been abandoned in most countries because of a high frequency of adverse effects ${ }^{3}$ and replaced by nonionic monomers (iohexol, iopamidol, ioversol, iopramide, iomeprol, iopentol, and iobitridol), nonionic dimers (iodixanol), or ionic dimers (ioxaglate). ICMs are considered safe drugs, even if adverse reactions are reported in $1 \%^{4-6}$ to $3 \%{ }^{7}$ of administrations. This can be attributed to pharmacological toxicity (eg, nephrotoxicity) or hypersensitivity reactions, next to unrelated events. ${ }^{8}$ Hypersensitivity reactions can be subdivided into immediate (IHRs, occurring $\leq 1$ hour after administration) and nonimmediate (NIHRs, occurring from $>1$ hour to several days after administration) hypersensitivity reactions.' A minority of IHRs, and typically those with a severe clinical presentation, are considered to be IgE mediated and can be identified using skin testing. ${ }^{10-14}$ These reactions are classified as drug allergies. ${ }^{9}$ In contrast, non-IgE-mediated IHRs are generally considered to be nonallergic hypersensitivity reactions (formerly coined anaphylactoid reactions), resulting from nonspecific mast cell and/or basophil degranulation.' Some NIHRs appear to be T-cell-mediated and can likewise be diagnosed using skin testing. ${ }^{11-15}$

The frequency of IHRs is reported to be $0.7 \%$ to $3 \%$ in patients receiving nonionic ICMs with severe reactions occurring in $0.02 \%$ to $0.04 \%$. $^{7}$ Fatality rate (for both ionic and nonionic ICMs) is estimated to be in the range of 1 in $100,000^{16}$ to 1 in 10 million. 3 NIHRs are reported to occur in $0.5 \%$ to $3 \%$ of administrations and may include life-threatening severe NIHRs.

Risk factors for ICM drug hypersensitivity reactions (DHR) are poorly understood and include asthma, ${ }^{17}$ a previous severe reaction, ${ }^{3}$ and multiple exposures. ${ }^{5}$ However, up to $34 \%{ }^{18,19}$ of reactions to ICMs are reported to occur on the first exposure suggesting a nonallergic nature in a subset of reactions and/or previous exposure to a hitherto unidentified sensitizing agent.

Most adverse effects result from intravascular administration, although case reports of extravascular administration, including oral administration, associated with severe or life-threatening reactions have been reported. ${ }^{1,20,21}$

Currently, in patients with a possible DHR, multiple strategies exist including avoidance of all ICMs, premedication on readministration $^{8}$ although controversial, or, as recommended by the international consensus on drug allergy, ${ }^{9}$ a drug allergy workup to identify a potential drug allergy and cross-reacting drugs. $7,9,11$ However, whether the latter approach using skin testing alone can also propose safe alternatives remains uncertain. Only few small series evaluated the negative predictive value
(NPV) of skin testing in both IHRs ${ }^{12,14,22-25}$ and NIHRs. ${ }^{12,15,24,26-28}$ A recent meta-analysis ${ }^{29}$ indicated that in patients with an initial IHR, 6 of $116(7.1 \%, 95 \%$ confidence interval $[\mathrm{CI}] 3.6 \%$ to $13.6 \%$ ) patients did not tolerate reexposure with a negatively skin-tested ICM, with most reactions being similar or milder and without premedication use in most studies. ${ }^{12,14,23,25}$ In NIHRs, a pooled 66 of 209 (34.5\%, $95 \%$ CI $18.7 \%$ to $54.8 \%$ ) re-exposed patients reacted, ${ }^{29}$ suggesting a lower NPV of skin testing in NIHRs compared with IHRs. However, large uniform data series are lacking and multiple strategies exist including provocation testing in the absence of radiological examination. In this work, 597 patients evaluated with skin testing for a suspected ICM-mediated IHR or NIHR, the largest cohort to date, are presented.

\section{METHODS}

All patients referred to the Allergy Department of the University Hospital of Montpellier, France, from February 2001 to September 2014, with a compatible clinical history of an ICM-mediated DHR, were included. Patient data were stored in the Drug Allergy and Hypersensitivity Database, a case-control cohort. Clinical data were registered using the European Network of Drug Allergy (ENDA) drug allergy questionnaire before performing skin tests. ${ }^{\text {' }}$ DHR were classified as IHRs (occurring $\leq 1$ hour after ICM administration) and NIHRs (occurring $>1$ hour to 7 days after ICM exposure). ${ }^{8}$ The Ring and Messmer ${ }^{30}$ classification was used to classify the severity of IHRs. Patients presenting with isolated loss of consciousness were scored as grade 3 anaphylaxis, and those with isolated bronchospasm or malaise were considered as a separate group. Severe NIHRs were identified separately. ${ }^{8}$

Skin testing was typically performed with a set of 10 ICMs (amidotrizoate, ioxitalamate, iopamidol, iohexol, ioversol, iopromide, iomeprol, iobitridol, iodixanol, and ioxaglate) for optimal evaluation of potential cross-creativity, identification of alternatives, and to increase the likelihood of testing the culprit ICM in case this ICM was unknown, as previously described. ${ }^{12}$ In 193 patients, less than 10 ICMs were evaluated (in 47 patients only 1; in 65, 2-5; and in 70, 6-9 ICMs were tested). Briefly, skin prick tests (SPT) were performed with the undiluted commercially available solution, and in case of negativity, they were followed by intradermal tests (IDT). Evaluation for IHRs was performed 20 minutes after IDT at a 1:10 dilution, and for NIHRs or undetermined chronology (UC), delayed reading of SPT and IDT was performed. A subset of patients with an NIHR underwent IDT with the undiluted solution for optimal sensitivity (41/92 patients before September 2012, after which this was systematically performed in $25 / 25$ patients in accordance with Torres et $\mathrm{al}^{15}$ ). Immediate-reading SPT was considered positive if, after 15 minutes, the size of the wheal was at least $3 \mathrm{~mm}$ in diameter with surrounding erythema; for IDT, positivity was considered when the size of the initial wheal after injection of $0.05 \mathrm{~mL}$ increased by at least $3 \mathrm{~mm}$ in diameter with surrounding erythema after 20 minutes. ${ }^{9}$ Delayed reading of SPT and IDT was performed according to the international guidelines of the European Society of Contact Dermatitis. ${ }^{31}$ Patients left the department with the instruction that in the absence of radiological alternatives, a negatively skin-tested ICM could be used, that the predictive value of skin testing was uncertain, and the proximity of an anesthesiologist or physician accredited for advanced life support was recommended in case of an initial IHR. ${ }^{32}$ Re-exposure to any ICM was counteradvised in patients with a severe NIHR, regardless 
of the skin test result. Premedication (1 dose of a second-generation H1-antihistamine, twice a day, to be started 48 hours before the examination until the day of the examination) was only recommended for patients with all negative ICM skin tests with concurrent chronic urticaria and mast cell disorders (of note, no such patients were included in this study). For asthmatics, with all negative ICM skin tests presenting with isolated bronchospasm, optimal control of asthma and premedication with short-acting $\beta 2$ agonists was recommended. Oral corticosteroids were not advised as premedication.

Patients were contacted (by phone and/or post) between October 2013 and October 2014 and asked standardized questions: whether they had undergone an ICM administration after the allergy workup, what the outcome was, if they had taken premedication. Hospital medical records were evaluated for all patients for potential reexposure to ICM, and tolerance was verified and interpreted as negative if no adverse event was reported by the patient and medical records. Patients included in our initial study ${ }^{12}$ were recontacted.

Qualitative data were expressed in frequency and percent. Quantitative data were expressed as medians with range or interquartile range (IQR) with 25 th and 75 th percentiles (Q25-Q75 IQR, not normally distributed data assessed with a Shapiro-Wilk test). The NPV was defined as the proportion of patients with negative skin test results to at least $1 \mathrm{ICM}$ at first testing with tolerated re-exposure to a negatively skin-tested ICM out of the total number of patients re-exposed to a skin test negative ICM. Data were collected using the FileMaker Pro 7 software (Filemaker, Inc., Santa Clara, CA). The analysis was performed using SAS software (SAS University Edition, SAS Institute, Cary, NC) and R (R-3.2.3. pkg, R Foundation, Vienna, Austria). Comparisons for the qualitative data were carried out between groups using $\chi^{2}$ or Fisher's exact test for small samples. A risk factor analysis was performed using univariate and multivariate logistic regression, and odds ratios (OR) were expressed with 95\% CI.

\section{RESULTS}

\section{Patient characteristics and ICM skin testing}

Of the 597 patients included during the 13.5-year period, 387 $(64.8 \%)$ could be physically contacted and 210 (35.2\%) underwent a medical record search only. Four hundred and six patients $(68.0 \%)$ were females and the median age was 60 (range 13-92) years. Thirty-two (5.4\%) had asthma and $173(29.1 \%)$ were atopic (Table I).

Initial reactions were immediate in 423 (70.9\%), nonimmediate in $118(19.7 \%)$, and of UC in $56(9.4 \%)$ patients. For immediate reactions, $104(24.6 \%)$ were of grade 2, 100 $(23.6 \%)$ grades 3 or 4 , and $30(7.1 \%)$ had isolated respiratory symptoms (20) or malaise (10). Of the 118 NIHRs, 9 were identified as severe with possible/probable drug reaction, eosinophilia and systemic symptoms. ${ }^{33}$ In 80 (13.4\%) patients, the reaction occurred on first ICM exposure. The ICM implicated in the initial reaction was identified in 166 patients $(27.8 \%)$. The ICM most frequently involved was iomeprol (13.8\%), iopromide $(10.0 \%)$, and iodixanol $(7.5 \%)$, although information on their relative use is lacking.

Allergy workup demonstrated skin test positivity in 80 (13.4\%) patients: $56(70 \%)$ with an IHR, 20 (25\%) with an NIHR, and 4 with an UC. The median time interval between the reaction and skin testing was 52.0 months (IQR 4.5-215.9). The relationship between the chronology of skin test positivity
TABLE I. Patient characteristics $(n=597$

\begin{tabular}{lc}
\hline Information source, $\mathrm{n}(\%)$ & \\
Physically contacted and medical record search & $387(64.8)$ \\
By phone & $373(62.5)$ \\
By post & $14(2.4)$ \\
Medical record search only & $210(35.2)$ \\
Median age, $\mathrm{y}$ (range) & $60(13-92)$ \\
Female, $\mathrm{n}(\%)$ & $406(68.0)$ \\
Atopic, $\mathrm{n}(\%)$ & $173(29.1)$ \\
Asthma, $\mathrm{n}(\%)$ & $32(5.4)$ \\
Chronology of the initial DHR, $\mathrm{n}(\%)$ & \\
IHR & $423(70.9)$ \\
$\quad$ Grade 1 & $122(28.8)$ \\
Grade 2 & $104(24.6)$ \\
Grade 3/4 & $100(23.6)$ \\
$\quad$ Malaise and isolated bronchospasm & $30(7.1)$ \\
Other/not determined & $67(15.8)$ \\
NIHR & $118(19.7)$ \\
Severe (including possible/probable DRESS) & $9(7.6)$ \\
Undetermined chronology & $56(9.4)$ \\
Reaction on first exposure, $\mathrm{n}(\%)$ & $80(13.4)$ \\
Tolerated previous ICM exposure & $188(31.4)$ \\
Unknown previous exposure & $329(55.2)$ \\
Median delay in months between reaction & $52(4.5-215.9)$ \\
and tests (IQR) & \\
Skin test positivity, $\mathrm{n}(\%)$ & $80(13.4)$ \\
IHR & $56(70.0)$ \\
NIHR & $20(25.0)$ \\
Undetermined chronology & $4(5.0)$ \\
\hline$D H R, D$ \\
\hline
\end{tabular}

$D H R$, Drug hypersensitivity reaction; DRESS, drug reaction, eosinophilia and systemic symptoms; ICM, iodinated contrast medium; $I H R$, immediate hypersensitivity reaction; $I Q R$, interquartile range; $N I H R$, nonimmediate hypersensitivity reaction.

and clinical history was concordant in most cases $(82.5 \%)$, yet discordant in 7 of $80(8.8 \%)$ patients ( 4 with nonimmediate skin test positivity and an IHR and 3 with immediate skin test positivity with an NIHR), and undetermined in 7 of 80 . In 1 of 80 skin test positive cases, the initially suspected ICM was not retrieved amongst the positively skin-tested $\operatorname{ICM}(s)$ versus concordance in 37 of 80 and no data on the initial ICM in 42 of 80 patients.

\section{Patterns of cross-reactivity}

In the 54 patients with skin test positivity on immediate reading, 37 had only 1 positive skin test and 17 had crossreactivity (range $2-5$ of 10 tested molecules) (Fig 1 ). In the 26 cases with skin test positivity on delayed reading $(\mathrm{n}=23)$ or UC $(\mathrm{n}=3), 8$ had only a single positive test, whereas 18 had crossreactivity (range $2-10$ of 10 tested molecules) (Fig 2). If the culprit ICM was known and tested, concordance with skin test positivity was observed in 37 of $38(97.3 \%)$ patients: 24,12 , and 1 with immediate, nonimmediate, or UC of skin test positivity, respectively. Cross-reactivity was most frequently observed for iopromide and iomeprol (41.1\%) for immediate and ioversol and iomeprol $(55.5 \%)$ for nonimmediate test positivity and mostly within the group containing a common $N$-(2,3-dihydroxypropyl) carbamoyl side chain (iopromide, iomeprol, ioversol, iohexol, iodixanol). This pattern was more obvious in case of nonimmediate test positivity compared with immediate test positivity. 


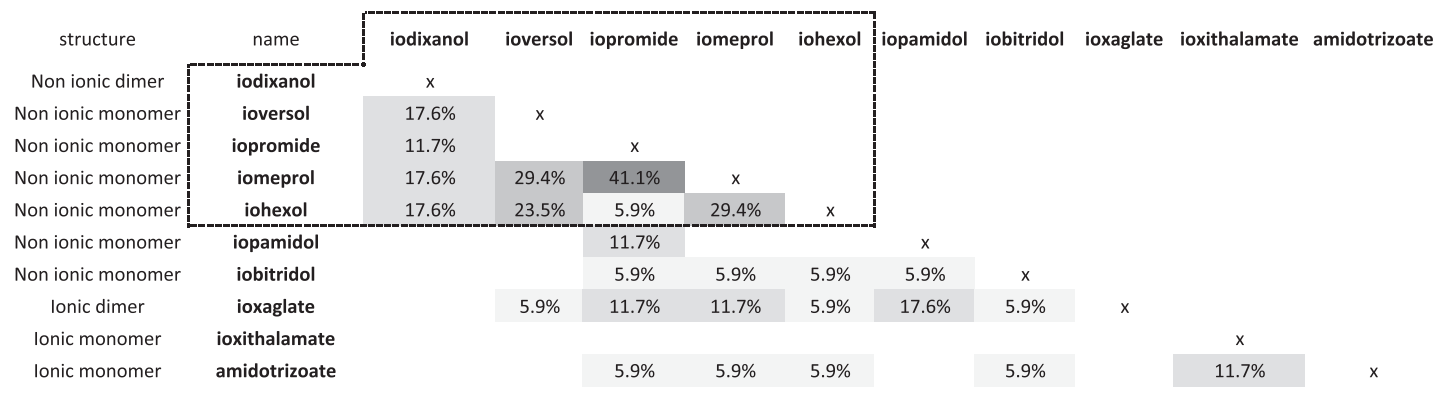

FIGURE 1. Skin test cross-reactivity in patients with IHR $(n=17)$. Cross-reactivity patterns for 17 patients with IHR and at least 2 immediately positive skin tests. Cross-reactivity was scored per pair, and the percentage of observed cross-reacting ICM pairs out of 17 possible pairs is demonstrated. ICMs with a common $\mathrm{N}$-(2,3-dihydroxypropyl) carbamoyl side chain are grouped with the dotted line. ICM, lodinated contrast medium; IHR, immediate hypersensitivity reaction.

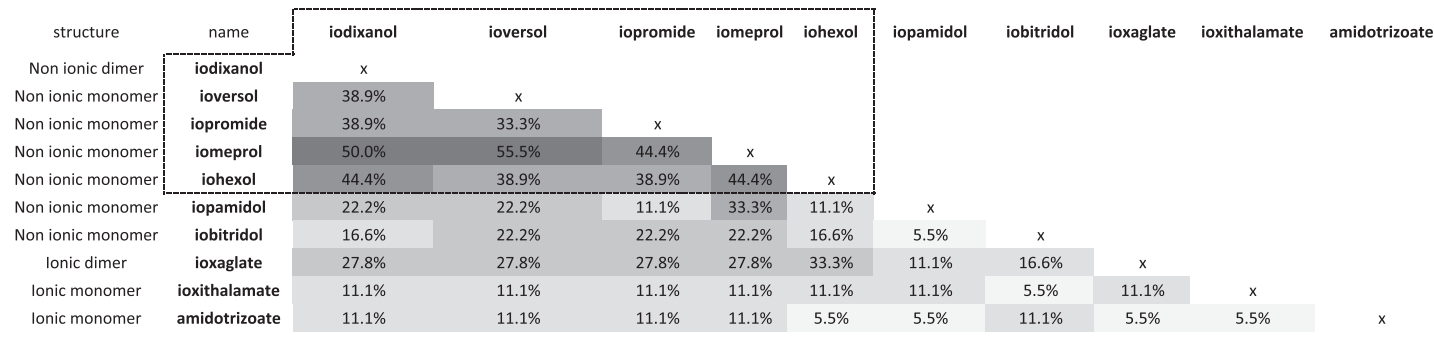

FIGURE 2. Skin test cross-reactivity in patients with NIHR $(n=18)$. Cross-reactivity patterns for 18 patients with NIHR and at least 2 positive skin tests. Cross-reactivity was scored per pair, and the percentage of observed cross-reacting ICM pairs out of 18 possible pairs is demonstrated. ICMs with a common $N$-(2,3-dihydroxypropyl) carbamoyl side chain are grouped with the dotted line. ICM, lodinated contrast medium; NIHR, nonimmediate hypersensitivity reaction.

\section{Patients challenged to a negatively skin-tested ICM}

In 233 of 597 (39.0\%), at least 1 challenge occurred with consistent tolerance in 217 of 233 patients (93.1\%), as outlined in Table II. Conversely, 16 of 233 patients re-exposed at least once with a negatively skin-tested ICM reacted at least once (6.9\%, $95 \%$ CI $3.6 \%$ to $10.1 \%$, Table III). Of these patients, 10 $(62.5 \%)$ had a history of an IHR and $6(37.5 \%)$ of an NIHR. Chronology of the reactions on challenge correlated with the initial reaction in all but 2 cases. One of the patients (no. 424) experienced a more severe reaction and 10 patients indicated that intensity on challenge was milder, 4 indicated that it was identical compared with the initial reaction, and in 1 no information regarding intensity was retrieved. In 4 patients, the initial and reexposed ICM were identical, and in 2, re-exposure with the same ICM did not always provoke a reaction, suggesting a nonimmunologically mediated mechanism. In 6 patients, multiple reactions were recorded with a noncrescendo evolution. On premedication for further exposures, 2 patients (nos. 528 and 269) reported the absence of adverse events. Patient 424 with an initial IHR after iomeprol and negative skin testing did not recall any re-exposures yet medical records stated a grade 3 anaphylaxis shortly after iohexol. Re-analysis of medical records, serum tryptase, and histamine quantifications, however, suggested an alternative diagnosis. The patient refused re-testing yet was included as a reactor on challenge.

In 17 of $80(21.2 \%)$ patients with at least 1 positive skin test, a challenge with a skin test negative ICM occurred (Fig 3). Challenges in these patients occurred significantly less frequently compared with 216 of 517 (41.8\%) challenges in patients with all negative skin tests $\left(P=.0005, \chi^{2}\right.$ test $)$. Of 17 patients, 16 tolerated the challenge (Tables II and III). The only patient reacting (no. 330) was rechallenged with the same ICM implicated in the initial reaction and for which skin testing on delayed reading was considered positive, and due to insufficient documentation retesting was suggested but refused. This patient could therefore also be considered being rechallenged to a skin test positive rather than negative ICM.

A challenge with the initially suspected ICM only occurred in 2 of 38 patients with 1 or more positive skin tests and a known initial ICM (patient 330 and patient 398, who had an initial maculopapular exanthema [MPE] 2 hours after iohexol and positive skin tests for multiple ICMs including iohexol, and was accidentally rechallenged with iohexol, next to iobitridol for which skin tests were negative, with tolerance for both-premedication status was unknown and iohexol rechallenge occurred at a later stage during chemotherapy courses). Conversely, in 18 of 216 $(8.3 \%)$ challenged patients with all negative skin tests, a rechallenge performed with the initially suspected ICM occurred with tolerance in 15 of $18(83.3 \%)$ patients.

Anaphylaxis grade 3 or 4 was the only factor associated with an increased risk for skin test positivity in a multivariate risk factor analysis including also previous exposure, multiple episodes suggestive of ICM DHR, sex, age at reaction, time between reaction and tests (OR 6.8, 95\% CI 3.2-14.5). A history of asthma was not identified as a risk factor for isolated bronchospasm during ICM injection although numbers were low. 
TABLE II. Challenges to negatively skin-tested ICM

\begin{tabular}{|c|c|c|c|c|c|c|c|}
\hline & \multirow[b]{2}{*}{ Total } & \multicolumn{3}{|c|}{ Initial chronology } & \multicolumn{3}{|c|}{ Skin test result } \\
\hline & & IHR & NIHR & UC & ST positive & ST negative & ST negative for a known culprit ICM \\
\hline Total & 597 & 423 & 118 & 56 & 80 & 517 & 125 \\
\hline Re-exposed, n (\%) & $233(39.0)$ & $172(40.6)$ & $43(36.4)$ & $18(32.1)$ & $17(21.2)$ & $216(41.8)$ & $51(69.7 \%)$ \\
\hline Tolerated, n (\%) & $217(93.1)$ & $162(94.2)$ & $37(86.0)$ & $18(100)$ & $16(94.1)$ & $201(93.1)$ & $43(84.3 \%)$ \\
\hline Not tolerated, n (\%) & $16(6.9)$ & $10(5.8)$ & $6(14.0)$ & $0(0)$ & $1 *(5.9)$ & $15(6.9)$ & $8(15.7 \%)$ \\
\hline
\end{tabular}

Number of re-exposed patients and percentage $(\%)$ from total or subgroup is shown.

$I C M$, Iodinated contrast medium; IHR, immediate drug hypersensitivity reaction; $M P E$, maculopapular exanthema; $N I H R$, nonimmediate drug hypersensitivity reaction; $S T$, skin test; $U C$, undetermined chronology.

*Patient 330 experienced an MPE on rechallenge with a skin test positive ICM.

\section{Premedication use}

In 150 re-exposed of 387 (38.8\%) physically contacted patients, $88(58.7 \%)$ reported no use of premedication, $31(20.7 \%)$ were not sure, and $31(20.7 \%)$ did use premedication although this was not systematically advised. Most premedication was prescribed by the treating physician at his or her own discretion. Reaction on re-exposure occurred in 13 of 150 patients $(8.7 \%)$ : $5(3.3 \%)$ without, $2(1.3 \%)$ with no certain information, and 6 $(4.0 \%)$ despite premedication, although only second-generation H1-antihistamines twice a day were administered. In 7 of 31 patients receiving premedication versus 4 of 88 patients not receiving premedication, a reaction on re-exposure occurred (19.4\% vs $5.7 \%, P=.01, \chi^{2}$ test).

\section{DISCUSSION}

Our group published the first study assessing the NPV of skin testing in ICM DHR. ${ }^{12}$ In 159 patients, 2 of 21 patients with positive skin tests and 27 of 99 patients with all negative skin tests were re-exposed. One patient with an initial NIHR described urticaria for 3 days but had a negative re-evaluation, using skin testing and a rechallenge with the same ICM as implicated in the previous reactions. Another patient developed an MPE, milder than the initial reaction. The NPV was estimated to be $96.6 \%$ (95\% CI $89.9 \%$ to $103.2 \%$ ), in line with the results of this larger study. Here, the overall NPV was $93.1 \%$ (95\% CI $89.1 \%$ to $96.0 \%$ ), and for IHRs and NIHRs $94.2 \%$ (95\% CI $89.6 \%$ to $97.2 \%$ ) and $86.1 \%$ (95\% CI $72.1 \%$ to $94.7 \%$ ), respectively. This result corroborates with a recent metaanalysis demonstrating a calculated NPV for IHRs $(\mathrm{n}=116)$ and NIHRs $(\mathrm{n}=209)$ of $94.8 \%$ (95\% CI 89.1-98.1) and $68.4 \%$ (95\% CI $61.6 \%$ to $74.7 \%$ ), respectively. ${ }^{29}$ Altogether, the NPV seems higher for patients with an IHR compared with an NIHR. In the largest previous series for patients with an NIHR, Torres et $\mathrm{al}^{15}$ observed 34 of $161(21 \%)$ positive skin tests and an additional $44(34 \%)$ patients with negative skin tests reacting on a provocation test. In our study, 20 of $118(17 \%)$ patients with an initial NIHR had positive skin tests with only 6 of $34(17.6 \%)$ reacting on re-exposure. All patients with an NIHR reacting on a challenge had been tested with the undiluted ICM, arguing against a sensitivity problem, and only a minority of patients $(51 / 118,43 \%)$ with an NIHR did not receive IDT with the undiluted culprit ICM. Next, we chose not to use a provocation test as a step-up procedure after negative skin testing, based on the fairly good NPV found in our initial report $^{12}$ and because we consider it would put patients at further risk for nonhypersensitivity adverse effects through unnecessary exposure (ie, in the absence of a radiological examination) to a potentially toxic ICM. Likewise, a provocation also does not discriminate between an allergic and nonallergic hypersensitivity reaction. Comparing the NPV between those with and without positive skin tests, we observed tolerance of challenge with a negatively skin-tested ICM in 16 of 17 (94.1\%) patients. The only patient reacting had presumably positive skin tests on delayed reading but refused further follow-up hampering a conclusive allergy workup, was rechallenged with this ICM, and experienced a new MPE. Omitting this patient, all 16 patients with at least 1 positive skin test tolerated a challenge with a skin test negative ICM. In patients with all negative skin tests, the majority $(201 / 215$, 93.1\%) tolerated re-exposure, and those reacting mostly had milder or identical reactions.

In our study, a lower proportion of patients (13.4\%) had positive skin tests compared with other groups $(29.1 \%$ to $64.7 \%^{11,15,18}$ ) using comparable skin test conditions. First, study populations might differ. Kim et al included only patients with an IHR with at least anaphylaxis grade 2, consistent with their lower incidence of reported IHRs $(0.016 \%$ or $104 / 632,513$ scans, ${ }^{18}$ compared with $0.8 \%$ to $1 \%$ in recent works ${ }^{4-6}$ ). In our study, patients with anaphylaxis grade 3 or 4 had a 6.8 -fold $(95 \%$ CI 3.2-14.5) increased risk for skin test positivity compared with other types of reactions. Interestingly, grade 2 anaphylaxis was not a significant risk factor, compared with milder reactions $(\mathrm{OR}=1.1,95 \%$ CI 0.4-2.6). Second, the delay between the initial reaction and testing might play a role. Brockow et $\mathrm{al}^{11}$ observed skin test positivity in up to $50 \%$ of patients tested within 6 months after the reaction, compared with only $22 \%$ of patients tested thereafter. Similarly, in our study, $25.9 \%$ (44/ 170) had positive skin tests within 6 months versus $8.4 \%$ (36/ 427, $P<.00001, \chi^{2}$ test) thereafter, arguing for skin testing early after the event (within 6 months) to increase sensitivity.

Sensitivity of skin testing for NIHRs to ICM has been demonstrated to be higher using a 1:1 dilution for IDT compared with a 1:10 dilution or patch testing. ${ }^{15}$ In our work, amongst 23 patients with skin tests positive on delayed reading, 3 were diagnosed only using IDT 1:1 and 3 had additional skin test positive ICMs using IDT 1:1, underscoring the findings by Torres et al. ${ }^{15}$

Although repeated exposure is considered a risk factor for IHRs, ${ }^{5}$ both IHRs and NIHRs were reported previously on first exposure in $23 \%{ }^{19}$ to $34 \% .{ }^{18}$ In our study, this was observed in $13.4 \%$ of the patients, and might be underestimated as in $55.2 \%$ of cases information on previous exposure was unknown. Interestingly, 12 of $80(15 \%)$ reactors on first exposure had positive skin tests with complete concordance (8/8) between culprit and positively tested ICM if known. Although we cannot exclude that skin testing would have been negative if performed 


\begin{tabular}{|c|c|c|c|c|c|c|c|c|c|c|c|c|c|}
\hline $\begin{array}{l}\text { Patient } \\
\text { number }\end{array}$ & Age/sex & Atopy & Initial reaction & IHR/NIHR & Grade & $\begin{array}{c}\text { Initial } \\
\text { culprit ICM }\end{array}$ & $\begin{array}{l}\text { Skin test } \\
\text { result (times } \\
\text { performed) }\end{array}$ & $\begin{array}{c}\text { Readministered } \\
\text { product }(\mathrm{n})\end{array}$ & Reaction-scheme & $\begin{array}{c}\text { Description (immediate/ } \\
\text { nonimmediate) }\end{array}$ & Grade & Premedication & $\begin{array}{c}\text { Concordance with } \\
\text { initial reaction }\end{array}$ \\
\hline 73 & $61 / \mathrm{F}$ & Yes & Loss of conscience & IHR & 3 & UD & neg & Iomeprol (n) & R R ... R & $\begin{array}{l}\text { Heat sensation and } \\
\text { impending doom, no } \\
\text { objective elements } \\
\text { (immediate) }\end{array}$ & I & No & Yes (less severe) \\
\hline 528 & $63 / \mathrm{M}$ & Yes & $\begin{array}{l}\text { General discomfort, } \\
\text { loss of conscience }\end{array}$ & IHR & 3 & $\begin{array}{r}\text { Ioxaglate or } \\
\text { ioversol }\end{array}$ & neg (2) & Iohexol (4)* & $00 \mathrm{R} 0$ & $\begin{array}{l}\text { Cough, heat sensation, } \\
\text { dyspnea, urticaria } \\
\text { (immediate) }\end{array}$ & 2 & No (since R yes) & Yes \\
\hline 464 & $44 / \mathrm{F}$ & No & AE, dyspnea, nausea & IHR & 2 & UD & neg & Iobitridol (4) & R R R R & $\begin{array}{l}\text { Identical reactions as first } \\
\text { episode yet less intense } \\
\text { (immediate) }\end{array}$ & 1 & $\begin{array}{c}\text { Yes (chronic } \\
\text { urticaria) }\end{array}$ & Yes (less severe) \\
\hline 284 & $71 / \mathrm{F}$ & Yes & $\begin{array}{c}\text { Heat sensation, } \\
\text { dyspnea }\end{array}$ & NIHR & & UD & neg & Iobitridol $(>5)$ & $\mathrm{R} R \ldots \mathrm{R}$ & $\begin{array}{l}\text { Altered taste, facial redness } \\
\text { for } 1 \mathrm{wk} \text { (immediate) }\end{array}$ & 1 & Yes & Yes (less severe) \\
\hline 358 & $39 / \mathrm{M}$ & No & $\begin{array}{c}\mathrm{U} \text {, general discomfort, } \\
\text { dyspnea }\end{array}$ & IHR & 2 & UD & neg & $\begin{array}{l}\text { Iomeprol (4), } \\
\text { iobitridol (1) }\end{array}$ & $000 \mathrm{R} 0$ & $\begin{array}{l}\text { Self-limiting rash } \\
\quad \text { (immediate) }\end{array}$ & 1 & UD & Yes (less severe) \\
\hline 46 & $56 / \mathrm{F}$ & Yes & Anaphylaxis & IHR & 2 & Iomeprol & neg & UD (n) & $\mathrm{R} R \ldots \mathrm{R}$ & $\begin{array}{l}\text { Redness upper body, self- } \\
\text { limiting }<1 \mathrm{~d} \text { (immediate) }\end{array}$ & 1 & Yes & $\begin{array}{c}\text { Yes (identical despite } \\
\text { premedication) }\end{array}$ \\
\hline 424 & $58 / \mathrm{F}$ & No & Anaphylaxis & IHR & 2 & Iomeprol & neg & Iohexol & $\mathrm{R}$ & Anaphylaxis $^{\ddagger}$ (immediate) & 3 & No & $\begin{array}{l}\text { Yes (more severe, } \\
\text { uncertain diagnosis) }\end{array}$ \\
\hline 588 & $70 / \mathrm{F}$ & UD & MPE or U & IHR & 1 & Iomeprol & neg & Iobitridol (1) & $\mathrm{R}$ & $\begin{array}{l}\text { Itch, possible urticaria } \\
\text { (immediate) }\end{array}$ & 1 & UD & Yes (less severe) \\
\hline 269 & $54 / \mathrm{F}$ & No & $\mathrm{U}$ & IHR & 1 & Iobitridol & neg & Iobitridol (5) & $000 \mathrm{R} 0$ & Cough (immediate) & 2 & $\begin{array}{l}\text { No before } \mathrm{R} \\
\text { (yes before } 0 \text { ) }\end{array}$ & Yes (UD) \\
\hline 199 & $22 / \mathrm{M}$ & Yes & MPE, AE & NIHR & & Iomeprol & neg & $\begin{array}{l}\text { Iomeprol (2), } \\
\text { iodixanol (1) }\end{array}$ & R R R & MPE (immediate) & I & Yes & Yes (less severe) \\
\hline 116 & $50 / \mathrm{F}$ & No & $\mathrm{U}$ & IHR & 1 & UD & neg & UD & $\mathrm{R}$ & $\begin{array}{l}\text { Redness back self-limiting } \\
\text { (immediate) }\end{array}$ & 1 & Yes & Yes (less severe) \\
\hline 293 & $70 / \mathrm{F}$ & Yes & EMP & IHR & 1 & UD & neg & $\begin{array}{l}\text { Ioversol (1), } \\
\text { iohexol (1) }\end{array}$ & R 0 & $\begin{array}{l}\text { Self-limiting facial redness/ } \\
\text { rash }<1 \mathrm{~h} \text { (immediate) }\end{array}$ & 1 & UD & Yes (less severe) \\
\hline 292 & $53 / \mathrm{F}$ & UD & $\mathrm{AE}$, dyspnea & NIHR & & Ioversol & neg (2) & Iopromide (1) & $\mathrm{R}$ & $\mathrm{AE}$ (nonimmediate) & & No & Yes (less severe) \\
\hline 176 & $32 / \mathrm{F}$ & No & MPE & NIHR & & UD & neg & Iomeprol (1) & $\mathrm{R}$ & MPE (nonimmediate) & & No & Yes \\
\hline 330 & $53 / \mathrm{F}$ & Yes & MPE & NIHR & & Iomeprol & $\operatorname{pos}^{\S}$ & Iomeprol (1) & $\mathrm{R}$ & $\begin{array}{l}\text { Erythema/AE } \\
\quad \text { (nonimmediate) }\end{array}$ & & UD & Yes \\
\hline 460 & $59 / \mathrm{F}$ & UD & MPE, AE & NIHR & & Iodixanol & neg & Iodixanol (2) & R R & MPE (nonimmediate) & & Yes & Yes (less severe) \\
\hline
\end{tabular}

$A E$, Angioedema; ICM, iodinated contrast medium; IHR, immediate drug hypersensitivity reaction; $N I H R$, nonimmediate drug hypersensitivity reaction, $M P E$, maculopapular exanthema; 0 , no reaction on rechallenge; $R$, reaction; $U$, urticarial; $U D$, undetermined.

* Discordance between patient response (iobitridol used) and medical record (iohexol used).

$\dagger$ Necessitating intravenous treatment (unspecified), no pre-existing asthma.

$\ddagger$ Anaphylaxis (bradycardia, hypotension) $10^{\prime}$ after iohexol, $45^{\prime}$ after propofol/sufentanil/cisatracurium. Analysis of medical records, serum tryptase, and histamine suggested an alternative (nonallergic) diagnosis. Follow-up refused.

$\S$ Unconfirmed and insufficiently documented skin test positivity for iodixanol, iomeprol, iohexol, ioversol, and iopromide. Patient refused follow-up. 


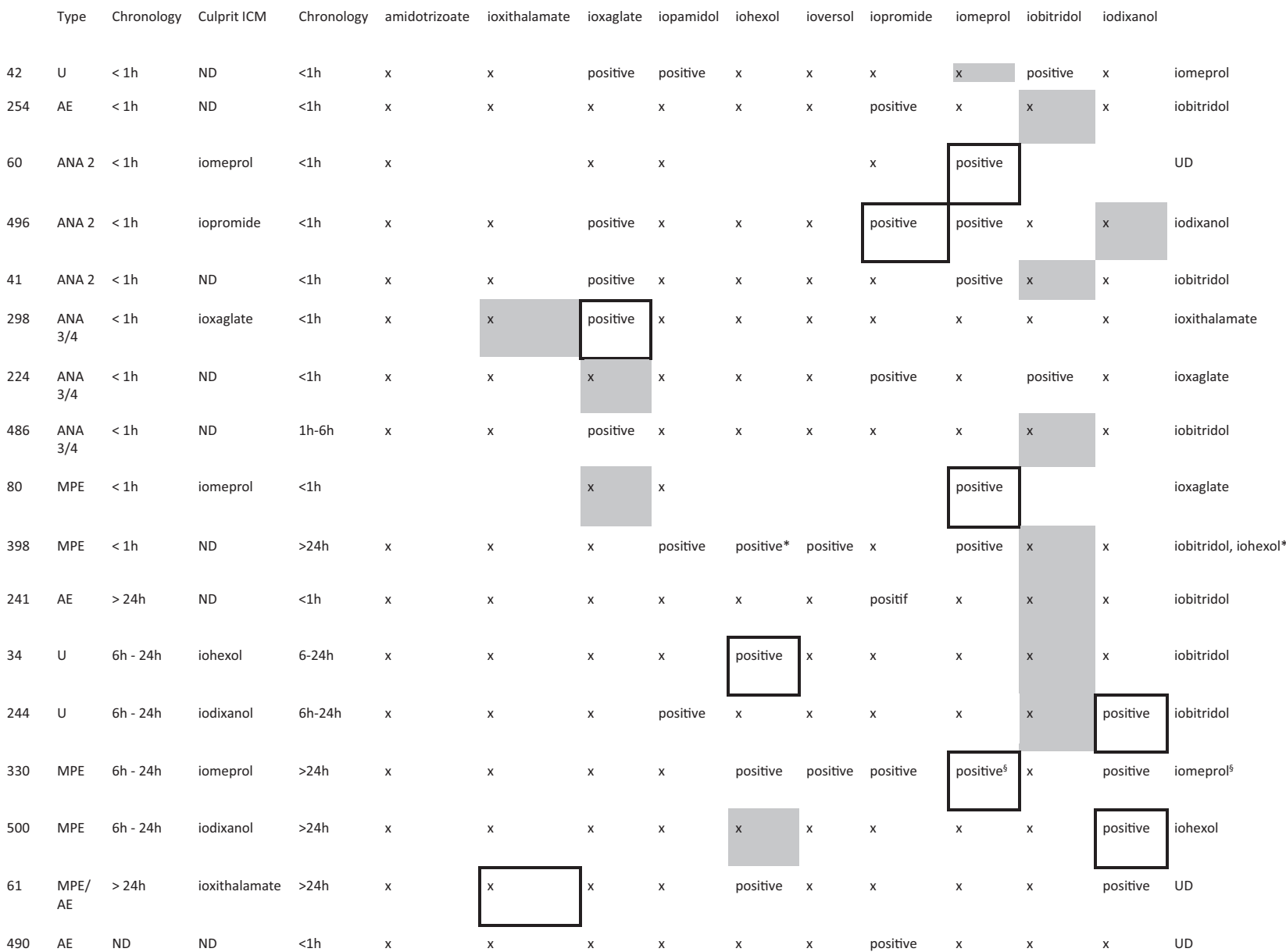

FIGURE 3. Patients with positive skin test results that were rechallenged. Skin-tested ICMs are marked with " $x$," "positive" represents positive skin test results, gray rechallenged and tolerated ICMs, and boxed the initial culprit ICM. *Patient 398 was rechallenged twice with iohexol ( 9 and 11 months after the initial reaction and perichemotherapy), without reactions. $\S$ Patient 330 was rechallenged with iomeprol despite putative positive skin tests on delayed reading, developed a second MPE reaction, but refused further follow-up. Patient 330 is the only patient reacting on rechallenge in this figure. AE, Angioedema; $A N A$, anaphylaxis; ICM, iodinated contrast medium; $M P E$, maculopapular exanthema; $N D$, not determined; $U D$, undetermined.

before the initial reaction, these results suggest an exposure to a hitherto unidentified sensitizing agent in at least a subset of patients experiencing a reaction on "first" exposure.

The presence of IgE-mediated ICM allergy has been long debated, yet the identification of specific anti-ICM IgE in the sera of patients, ${ }^{10,13}$ positive basophil activation tests, ${ }^{25}$ and immediate skin test positivity are arguments in favor. ${ }^{11}$ Our work adds to this idea and also enabled the study of crossreactivity patterns in 80 positively skin-tested patients. In IHRs, most patients reacted to only 1 ICM with a strong correlation with the suspected culprit ICM if this was known. In NIHRs, this correlation was also observed, yet a broader pattern of cross-reactivity was observed. Previously, Brockow et $\mathrm{al}^{11}$ suggested considerable cross-reactivity between iodixanol, iohexol, iopentol, iomeprol, and ioversol based on 36 patients with multiple skin test positivity. Lerondeau et $\mathrm{al}^{19}$ subdivided ICM into groups A (iodixanol, iohexol, iomeprol, ioversol, ioxithalamate, iopamidol), B (iobitridol, ioxaglate), and C (amidotrizoate) with frequent intragroup but infrequent intergroup cross-reactivity, especially in NIHRs, based on 65 patients with multiple skin test positivity. The $N$-(2,3-dihydroxypropyl) carbamoyl side chain was proposed as a common denominator for group A except for ioxithalamate and iopamidol. Our study in 35 patients with multiple positive skin tests indicates that this common side chain in iodixanol, iohexol, iomeprol, ioversol, and iopromide unifies the frequently cross-reacting ICMs, especially in NIHRs. We therefore propose to further define the previously proposed group $\mathrm{A}^{19}$ by including iopromide and excluding ioxithalamate and iopamidol, probably because the latter both lack the $N$-(2,3-dihydroxypropyl) carbamoyl side chain. Of note, specificity of skin testing for ICM is well established, ${ }^{7}$ yet we cannot exclude that the nonspecific false positive skin test results in a subset of patients because specific IgE determination or isolation of drug-specific $\mathrm{T}$ cells was not performed. 
In our work, we could not identify a reaction threshold for IHRs below which testing would be needless, because 4 and 3 patients with IHRs presenting with isolated urticaria or angioedema respectively had positive skin test results. Because grade 1 IHRs to ICM are generally considered to be mediated by nonspecific histamine release and sensitization profile in the general population is unknown, we cannot explain skin test positivity in these patients. We could argue that clinical history is not always certain and some patients might underestimate their symptoms. However, the probability for skin test positivity clearly increased with increasing severity.

Premedication with steroids, antihistamines, and other drugs, alone or in combination, is widely used before injection of ICM. ${ }^{34}$ However, few studies have evaluated the efficacy of premedication to reduce the number of ICM-mediated DHR and most are hampered by methodological concerns (reviewed in the paper by Tramèr et $\mathrm{al}^{34}$ ) such as the absence of a comparator arm, ${ }^{35}$ retrospective setup, ${ }^{36}$ the lack of focus on patients with a previous ${ }^{37}$ or severe reaction, ${ }^{38}$ or the use of the mostly abandoned ionic ICM. ${ }^{39,40}$ Lasser et $\mathrm{al}^{37}$ reported an overall reduction in reactions on steroid premedication in an unselected population $(1.7 \%$ for the premedicated vs $4.9 \%$ for the placebo group, $P=.005)$, but observed no significant reduction in patients experiencing moderate-to-severe IHRs or in patients who had a history of an ICM-mediated IHR ( $8.8 \%$ for the premedicated vs $9.7 \%$ for the placebo group). Moreover, so-called "breakthrough" reactions occurring despite premedication in untested patients, often severe, are widely reported. $25,37,41,42$ Therefore, physicians should not rely on the efficacy of premedication, ${ }^{43}$ although perhaps in patients without evidence for an IgE-mediated IHR, premedication might reduce symptoms from nonallergic IHR as illustrated by some cases in this work. Our work provides evidence for an alternative approach to perform skin testing in patients with a clinical reaction resembling allergy (IHR or NIHR) and to use a negatively skintested ICM afterward, with only exceptional use of premedication, in a safe environment, and if strictly necessary in the context of imaging. A significantly larger proportion of patients receiving premedication reacted compared with those not receiving premedication $\left(19.4 \%\right.$ vs $5.7 \%, P=.01, \chi^{2}$ test). Premedication, here $\mathrm{H} 1$-antihistamines twice a day, did not prevent reactions completely. However, this study was not designed to evaluate the efficacy of premedication and we can only speculate if a more extensive premedication regimen or none at all would have given different results. In addition, because premedication was not systematically advised at the allergy consultation yet prescribed by some treating physicians, a selection bias cannot be excluded.

In this study, the percentage of re-exposed patients (233/597, $39.0 \%$ ) was comparable to that in our previous report $(29 / 159$, $37.1 \%) .{ }^{12}$ Two patients were re-exposed accidentally to a positively skin-tested ICM suggesting suboptimal dissemination of information on allergy (or no allergy) toward patients and health care workers.

This study has certain limitations. Because information on culprit and/or re-exposed ICM was lacking in many patients, we cannot rule out that many challenges in this subgroup also occurred with a different ICM than the initial culprit ICM and possibly overestimates the NPV. Next, this work was not intended to evaluate the use (or not) of premedication as such, and we cannot exclude that premedication can be useful to reduce symptoms in patients suffering from nonallergic hypersensitivity reactions.
In conclusion, our study demonstrates that skin testing for suspected ICM-mediated DHR is useful and can identify safe alternatives for further real-life setting injection of ICM. Skin testing is preferred early (1-6 months) after the event. In case of skin-test confirmed ICM drug allergy, a challenge with a skin test negative ICM was considered uneventful. In those with all negative skin test results, only a small subset of patients experienced symptoms on re-exposure, often milder, especially when re-exposed to the same ICM. The data support an allergologistdriven, clinical history-, and skin-test-based approach to guide ICM re-exposure, without the need for provocation testing outside an imaging context. Prospective studies are warranted to confirm these observations.

\section{REFERENCES}

1. Singh J, Daftary A. Iodinated contrast media and their adverse reactions. J Nucl Med Technol 2008;36:69-74-7.

2. Lieberman P, Siegle RL, Treadwell G. Radiocontrast reactions. Clin Rev Allergy 1986;4:229-45.

3. Katayama H, Yamaguchi K, Kozuka T, Takashima T, Seez P, Matsuura K. Adverse reactions to ionic and nonionic contrast media. A report from the Japanese Committee on the Safety of Contrast Media. Radiology 1990;175: 621-8.

4. Farolfi A, Della Luna C, Ragazzini A, Carretta E, Gentili N, Casadei C, et al. Taxanes as a risk factor for acute adverse reactions to iodinated contrast media in cancer patients. Oncologist 2014;19:823-8.

5. Fujiwara N, Tateishi R, Akahane M, Taguri M, Minami T, Mikami S, et al. Changes in risk of immediate adverse reactions to iodinated contrast media by repeated administrations in patients with hepatocellular carcinoma. PLoS One 2013;8:e76018.

6. Pradubpongsa P, Dhana N, Jongjarearnprasert K, Janpanich S, Thongngarm T. Adverse reactions to iodinated contrast media: prevalence, risk factors and outcome-the results of a 3-year period. Asian Pac J Allergy Immunol 2013;31: 299-306.

7. Brockow K, Sánchez-Borges M. Hypersensitivity to contrast media and dyes. Immunol Allergy Clin North Am 2014;34:547-64, viii.

8. Brockow K, Christiansen C, Kanny G, Clément O, Barbaud A, Bircher A, et al. Management of hypersensitivity reactions to iodinated contrast media. Allergy 2005;60:150-8.

9. Demoly P, Adkinson NF, Brockow K, Castells M, Chiriac AM, Greenberger PA, et al. International consensus on drug allergy. Allergy 2014;69:420-37.

10. Laroche D, Aimone-Gastin I, Dubois F, Huet H, Gérard P, Vergnaud MC, et al. Mechanisms of severe, immediate reactions to iodinated contrast material. Radiology 1998;209:183-90.

11. Brockow K, Romano A, Aberer W, Bircher AJ, Barbaud A, Bonadonna P, et al. Skin testing in patients with hypersensitivity reactions to iodinated contrast media—a European multicenter study. Allergy 2009;64:234-41.

12. Caimmi S, Benyahia B, Suau D, Bousquet-Rouanet L, Caimmi D, Bousquet P-J, et al. Clinical value of negative skin tests to iodinated contrast media. Clin Exp Allergy 2010;40:805-10.

13. Mita H, Tadokoro K, Akiyama K. Detection of IgE antibody to a radiocontrast medium. Allergy 1998;53:1133-40.

14. Prieto-García A, Tomás M, Pineda R, Tornero P, Herrero T, Fuentes V, et al. Skin test-positive immediate hypersensitivity reaction to iodinated contrast media: the role of controlled challenge testing. J Investig Allergol Clin Immunol 2013;23:183-9

15. Torres MJ, Gomez F, Doña I, Rosado A, Mayorga C, Garcia I, et al. Diagnostic evaluation of patients with nonimmediate cutaneous hypersensitivity reactions to iodinated contrast media. Allergy 2012;67:929-35.

16. Caro JJ, Trindade E, McGregor M. The risks of death and of severe nonfatal reactions with high- vs low-osmolality contrast media: a meta-analysis. AJR Am J Roentgenol 1991;156:825-32.

17. Lang DM, Alpern MB, Visintainer PF, Smith ST. Increased risk for anaphylactoid reaction from contrast media in patients on beta-adrenergic blockers or with asthma. Ann Intern Med 1991;115:270-6.

18. Kim M-H, Lee S-Y, Lee S-E, Yang M-S, Jung J-W, Park CM, et al. Anaphylaxis to iodinated contrast media: clinical characteristics related with development of anaphylactic shock. PLoS One 2014;9:e100154.

19. Lerondeau B, Trechot P, Waton J, Poreaux C, Luc A, Schmutz J-L, et al. Analysis of cross-reactivity among radiocontrast media in 97 hypersensitivity reactions. J Allergy Clin Immunol 2016;137:633-635.e4. 
20. Davis PL. Anaphylactoid reactions to the nonvascular administration of water-soluble iodinated contrast media. AJR Am J Roentgenol 2015;204: 1140-5.

21. Pasternak JJ, Williamson EE. Clinical pharmacology, uses, and adverse reactions of iodinated contrast agents: a primer for the non-radiologist. Mayo Clin Proc 2012;87:390-402.

22. Dewachter P, Laroche D, Mouton-Faivre C, Bloch-Morot E, Cercueil J-P, Metge L, et al. Immediate reactions following iodinated contrast media injection: a study of 38 cases. Eur J Radiol 2011;77:495-501.

23. Salas M, Gomez F, Fernandez TD, Doña I, Aranda A, Ariza A, et al. Diagnosis of immediate hypersensitivity reactions to radiocontrast media. Allergy 2013; 68:1203-6.

24. Scherer K, Harr T, Bach S, Bircher AJ. The role of iodine in hypersensitivity reactions to radio contrast media. Clin Exp Allergy 2010;40:468-75.

25. Trcka J, Schmidt C, Seitz CS, Bröcker E-B, Gross GE, Trautmann A. Anaphylaxis to iodinated contrast material: nonallergic hypersensitivity or IgEmediated allergy? AJR Am J Roentgenol 2008;190:666-70.

26. Hasdenteufel F, Waton J, Cordebar V, Studer M, Collignon O, Luyasu S, et al. Delayed hypersensitivity reactions caused by iodixanol: an assessment of crossreactivity in 22 patients. J Allergy Clin Immunol 2011;128:1356-7.

27. Seitz CS, Pfeuffer P, Raith P, Bröcker E-B, Trautmann A. Radiocontrast mediaassociated exanthema: identification of cross-reactivity and tolerability by allergologic testing. Eur J Radiol 2009:72:167-71.

28. Vernassiere C, Trechot P, Commun N, Schmutz J-L, Barbaud A. Low negative predictive value of skin tests in investigating delayed reactions to radio-contrast media. Contact Dermatitis 2004;50:359-66.

29. Yoon SH, Lee S-Y, Kang H-R, Kim J-Y, Hahn S, Park CM, et al. Skin tests in patients with hypersensitivity reaction to iodinated contrast media: a metaanalysis. Allergy 2015;70:625-37.

30. Ring J, Messmer K. Incidence and severity of anaphylactoid reactions to colloid volume substitutes. Lancet 1977;1:466-9.

31. Johansen JD, Aalto-Korte K, Agner T, Andersen KE, Bircher A, Bruze M, et al European Society of Contact Dermatitis guideline for diagnostic patch testingrecommendations on best practice. Contact Dermatitis 2015;73:195-221.
32. Kvedariene V, Martins P, Rouanet L, Demoly P. Diagnosis of iodinated contrast media hypersensitivity: results of a 6-year period. Clin Exp Allergy 2006;36:1072-7.

33. Kardaun SH, Sidoroff A, Valeyrie-Allanore L, Halevy S, Davidovici BB, Mockenhaupt M, et al. Variability in the clinical pattern of cutaneous sideeffects of drugs with systemic symptoms: does a DRESS syndrome really exist? Br J Dermatol 2007;156:609-11.

34. Tramèr MR, von Elm E, Loubeyre $\mathrm{P}$, Hauser C. Pharmacological prevention of serious anaphylactic reactions due to iodinated contrast media: systematic review. BMJ 2006;333:675.

35. Greenberger PA, Patterson R. The prevention of immediate generalized reactions to radiocontrast media in high-risk patients. J Allergy Clin Immunol 1991;87:867-72.

36. Wolf GL, Mishkin MM, Roux SG, Halpern EF, Gottlieb J, Zimmerman J, et al. Comparison of the rates of adverse drug reactions. Ionic contrast agents, ionic agents combined with steroids, and nonionic agents. Invest Radiol 1991;26:404-10.

37. Lasser EC, Berry CC, Mishkin MM, Williamson B, Zheutlin N, Silverman JM. Pretreatment with corticosteroids to prevent adverse reactions to nonionic contrast media. AJR Am J Roentgenol 1994;162:523-6.

38. Kolbe AB, Hartman RP, Hoskin TL, Carter RE, Maddox DE, Hunt CH, et al. Premedication of patients for prior urticarial reaction to iodinated contrast medium. Abdom Imaging 2014;39:432-7.

39. Lasser EC, Berry CC, Talner LB, Santini LC, Lang EK, Gerber FH, et al Pretreatment with corticosteroids to alleviate reactions to intravenous contrast material. N Engl J Med 1987;317:845-9.

40. Lasser EC, Berry CC, Talner LB, Santini LC, Lang EK, Gerber FH, et al. Protective effects of corticosteroids in contrast material anaphylaxis. Invest Radiol 1988;23(Suppl 1):S193-4.

41. Williams AN, Kelso JM. Radiocontrast-induced anaphylaxis despite pretreatment and use of iso-osmolar contrast. Ann Allergy Asthma Immunol 2007;99:467-8.

42. Worthley DL, Gillis D, Kette F, Smith W. Radiocontrast anaphylaxis with failure of premedication. Intern Med J 2005;35:58-60.

43. ACR Committee on Drugs and Contrast MediaACR Manual on Contrast Media Version 10.3 2017. Available from: https:/www.acr.org/ /media/ACR/Documents/ PDF/QualitySafety/Resources/Contrast-Manual/Contrast_Media.pdf?db=web. Accessed August 14, 2017. 